\title{
The estimates on the energy functional of an elliptic system with Neumann boundary conditions
}

Jing Zeng*

\section{"Correspondence:}

zengjing@fjnu.edu.cn

School of Mathematics and

Computer Sciences, Fujian Normal

University, Fuzhou, 350007, P.R.

China

\begin{abstract}
We consider an elliptic system of the form $-\varepsilon^{2} \Delta u+u=f(v),-\varepsilon^{2} \Delta v+v=g(u)$ in $\Omega$ with Neumann boundary conditions, where $\Omega$ is a $C^{2}$ domain in $\mathbb{R}^{N}, f$ and $g$ are nonlinearities having superlinear and subcritical growth at infinity. We prove the existence of nonconstant positive solutions of the system, and estimate the energy functional on a configuration space $\bar{H}$ by a different technique, which is an important step in the proof of the solution's concentrative property. We conclude that the least energy solutions of the system concentrate at the point of boundary, which maximizes the mean curvature of $\partial \Omega$.
\end{abstract}

Keywords: elliptic system; estimates; energy functional

\section{Introduction}

We are concerned with the following singularly perturbed system with Neumann conditions:

$$
\left\{\begin{array}{l}
-\varepsilon^{2} \Delta u+u=g(v), \quad \text { in } \Omega, \\
-\varepsilon^{2} \Delta v+v=f(u), \quad u, v>0, \text { in } \Omega, \\
\frac{\partial u}{\partial n}=\frac{\partial v}{\partial n}=0, \quad \text { on } \partial \Omega,
\end{array}\right.
$$

where $\varepsilon>0$ is a small parameter, $\Omega$ is a $C^{2}$ bounded domain in $\mathbb{R}^{N}(N \geq 3), 0 \in \Omega$. $f$ and $g$ are nonlinearities having superlinear and subcritical growth at infinity.

Problem (1.1) arises in many applied models concerning biological pattern formations. For example, the steady states in the Keller-Segel model, the Gierer-Meinhardt model, see $[1,2]$ for more details. Problem (1.1) has been studied extensively for last twenty years. The motivation for the study of such a problem goes back to the pioneering work of $[2,3]$ concerning the scalar case (single equation),

$$
\left\{\begin{array}{l}
-\varepsilon^{2} \Delta u+u=u^{p}, \quad u>0, \text { in } \Omega, \\
\frac{\partial u}{\partial n}=0, \quad \text { on } \partial \Omega .
\end{array}\right.
$$

They proved a priori estimates, existence of least energy solutions and the concentrative properties of the solution. Furthermore, in $[4,5], \mathrm{Ni}$ and Takagi proved the existence of

(c) 2013 Zeng; licensee Springer. This is an Open Access article distributed under the terms of the Creative Commons Attribution License (http://creativecommons.org/licenses/by/2.0), which permits unrestricted use, distribution, and reproduction in any medium, provided the original work is properly cited. 
a nontrivial solution $u_{\varepsilon}$ to problem (1.2) for $\varepsilon$ small enough. They showed that $u_{\varepsilon}$ attains its maximum value at a point $P_{\varepsilon} \in \partial \Omega$, and the subsequences of $P_{\varepsilon}$ converge to $P$, which is the maximum point of mean curvature on $\partial \Omega$.

The subject was studied by many authors for both Neumann and Dirichlet boundary conditions. There are many well-known results about (1.2). Del Pino and Felmer in [6] introduced shorter and more elementary arguments with respect to those in [4, 7]. Wang in [8] obtained multiple solutions of (1.2) by using Ljusternik-Schnirelman method. In [9], Grossi et al., obtained a solution of (1.2) with $k$ maxima points, $k$ is a given positive integer. We refer the reader to [10-14] for further references.

As far as we know, Avial and Yang [15] were the first to approach the singularly perturbed system (1.1) with Neumann boundary conditions; they considered (1.1) with special nonlinearities $f(s)=s^{p-1}, g(s)=s^{q-1}(2<p, q<2 N / N-2)$. By means of a dual variational formulation, they proved that there exist nontrivial positive solutions $u_{\varepsilon}$ and $v_{\varepsilon}$ in $C^{2}(\bar{\Omega})$, which have global maximum point at different points.

A more direct approach was proposed in [16-18]. In these papers, the authors extend the idea, which is introduced by Del Pino and Felmer in [6], to system (1.1). In [18], Pistoia and Ramos proved the least energy solutions of system (1.1) concentrate at a point of the boundary, which maximizes the mean curvature of the boundary of $\Omega$. Pistoia and Ramos [19] consider system (1.1) with Dirichlet boundary condition, they proved the existence of the least energy solutions. The solutions are concentrated, as $\varepsilon$ goes to zero, at a point of $\Omega$, which is maximized in distance to the boundary of $\Omega$.

Let us recall the idea mentioned in [3-5], their proof based on the well-known result of Gidas et al. [20], that is, the uniqueness solution of the equation

$$
\left\{\begin{array}{l}
-\Delta w+w=w^{p}, \quad w>0, \text { in } \mathbb{R}^{N}, \\
w(0)=\max _{y \in \mathbb{R}^{N}} w(y), \quad w \rightarrow 0, \text { as }|y| \rightarrow+\infty,
\end{array}\right.
$$

here $w$ is radially symmetric and is strictly decreasing, and $w^{\prime}(r)<0$ for $r>0$. But the uniqueness result for the following system corresponding to (1.1) is not known.

$$
\left\{\begin{array}{l}
-\Delta u+u=g(v), \quad \text { in } \mathbb{R}^{N}, \\
-\Delta v+v=f(u), \quad \text { in } \mathbb{R}^{N}, \\
u, v>0, \quad \text { in } \mathbb{R}^{N} .
\end{array}\right.
$$

Besides, it is known that the underlying minimax theorem associated to ground-state level of (1.1) is an infinite-dimensional linking, this is in contrast with (1.2). We refer the reader to $[18,21]$ for more details on this.

In this paper, we prove the existence of nonconstant positive solutions $u_{\varepsilon}, v_{\varepsilon} \in C^{2}(\Omega) \cap$ $H^{1}(\Omega)$ of system (1.1), and estimate the energy functional of (1.1) on the configuration space $\bar{H}$ (defined in Section 2) by a different technique, which is compared with [18]. This estimation is an important step in the proof of $H\left(P_{0}\right)=\max _{P \in \partial \Omega} H(P)$, where $H(P)$ denotes the mean curvature of $\partial \Omega$ at the boundary point $P$. We conclude the least energy solutions of system (1.1), concentrated at the point of boundary, which maximizes the mean curvature of the boundary of $\Omega$. 


\section{Statement of main results}

The assumption to $f, g \in C^{1}\left(\mathbb{R}^{N}\right)$ is a typical superlinear subcritical one, as in [18], we assume that the following holds.

$\left(\mathrm{S}_{1}\right) f(s)=g(s)=0$, for $s \leq 0 . f(0)=0=f^{\prime}(0), g(0)=0=g^{\prime}(0)$. There exist two real numbers $l_{1}, l_{2}>0,2<p, q<2 N /(N-2)$ such that

$$
\lim _{|s| \rightarrow \infty} f^{\prime}(s)|s|^{2-p}=l_{1}, \quad \lim _{|s| \rightarrow \infty} g^{\prime}(s)|s|^{2-q}=l_{2} .
$$

$\left(\mathrm{S}_{2}\right)$ For some $\delta>0, s \in \mathbb{R}, s \neq 0$,

$$
f^{\prime}(s) s^{2} \geq(1+\delta) f(s) s>0, \quad g^{\prime}(s) s^{2} \geq(1+\delta) g(s) s>0 .
$$

$\left(\mathrm{S}_{3}\right) f^{2}(s) \leq 2 f^{\prime}(s) F(s), g^{2}(s) \leq 2 g^{\prime}(s) G(s)$, for $s \in \mathbb{R}, s \neq 0$, where $F(s)=\int_{0}^{s} f(t) d t, G(s)=$ $\int_{0}^{s} g(t) d t$.

Remark 2.1 Examples of nonlinearities satisfying $\left(\mathrm{S}_{1}\right)-\left(\mathrm{S}_{3}\right)$ are

$$
f(s)=a_{1}|s|^{b_{1}}+|s|^{p-1}, \quad g(s)=a_{2}|s|^{b_{2}}+|s|^{q-1},
$$

where $a_{1}, a_{2}>0,2<b_{1} \leq p-1,2<b_{2} \leq q-1,2<p, q<2 N /(N-2)$.

We should point out that $\left(\mathrm{S}_{1}\right)-\left(\mathrm{S}_{3}\right)$ are the natural extension of the assumptions for the scalar case (single equation). Let us recall the assumptions on single equation such as (1.2). Assume that $f: \mathbb{R} \rightarrow \mathbb{R}$ is continuous and satisfies the following structure assumptions.

(f $\left.f_{1}\right) f(t)=0$ for $t \leq 0$ and $f(t)=o(t)$ near $t=0 . f(t)=O\left(t^{s}\right)$ as $t \rightarrow \infty$, for some $1<s<$ $(N+2) /(N-2)$ if $N>=3$, and $s>1$ if $N=1,2$.

$\left(\mathrm{f}_{2}\right)$ There exists a constant $\theta>2$ such that $\theta F(t) \leq t f(t)$ for $t \geq 0$, in which $F(t)=\int_{0}^{t} f(s) d s$.

$\left(\mathrm{f}_{3}\right)$ The function $t \rightarrow f(t) / t$ is strictly increasing.

Remark 2.2 Assumption $\left(\mathrm{S}_{1}\right)$ is the 'system edition' of $\left(\mathrm{f}_{1}\right)$. $\left(\mathrm{f}_{2}\right)$ is the famous AmbrosettiRabinowitz superlinear condition [22], which has appeared in most of studies for superlinear problems. In fact, it implies that the super-quadratic condition on $F(t)$. It has been used in a crucial way not only in establishing the mountain-pass geometry of the functional, but also in obtaining bounds of (PS) sequences. Assumption $\left(\mathrm{S}_{2}\right)$ implies that $f(s) s \geq(2+\delta) F(s)>0, g(s) s \geq(2+\delta) G(s)>0$, which play a important roll in the proof of the existence of system's solutions. So it is the 'system edition' of $\left(\mathrm{f}_{2}\right)$.

Without loss of generally, we may assume that $0 \in \Omega$. By the following rescaling:

$$
z=\varepsilon \mathcal{X}, \quad x \in \Omega_{\varepsilon}:=\{\varepsilon x \in \Omega\},
$$

equation (1.1) becomes

$$
\left\{\begin{array}{l}
-\Delta u+u=g(v), \quad \text { in } \Omega_{\varepsilon}, \\
-\Delta v+v=f(u), \quad u, v>0 \text { in } \Omega_{\varepsilon}, \\
\frac{\partial u}{\partial n}=\frac{\partial v}{\partial n}=0, \quad \text { on } \partial \Omega_{\varepsilon} .
\end{array}\right.
$$


To simplify the notations, we define $\Omega:=\Omega_{\varepsilon}$. Associated with (2.1) is the energy functional

$$
I(u, v)=\int_{\Omega}(\langle\nabla u, \nabla v\rangle+u v)-\int_{\Omega}(F(u)+G(v)),
$$

(2.2) is a $C^{2}$ functional defined over the Hilbert space $H:=H^{1}(\Omega) \times H^{1}(\Omega)$.

We define the norm

$$
\|(u, v)\|^{2}:=\|u\|^{2}+\|v\|^{2}
$$

where $\|u\|^{2}=\int_{\Omega}\left(|\nabla u|^{2}+u^{2}\right)$.

It can be observed that the following orthogonal splitting holds: $H=H_{-} \oplus H_{+}$, here, $H_{-}:=\left\{(\phi,-\phi), \phi \in H^{1}(\Omega)\right\}, H_{+}:=\left\{(\phi, \phi), \phi \in H^{1}(\Omega)\right\}$. We set $\widetilde{H}=\left\{t \cdot(u, v) \mid t \in \mathbb{R}^{+}, u, v \in\right.$ $\left.H^{1}(\Omega), u \neq-v\right\}, \bar{H}=H_{-} \oplus \widetilde{H}$.

Theorem 2.3 Assume $\left(\mathrm{S}_{1}\right)-\left(\mathrm{S}_{3}\right)$, then there exists $\varepsilon_{0}>0$, such that for any $0<\varepsilon<\varepsilon_{0}$, system (1.1) has nonconstant positive solutions $u_{\varepsilon}, v_{\varepsilon} \in C^{2}(\Omega) \cap H^{1}(\Omega)$. If $u_{\varepsilon} \neq-v_{\varepsilon}$, the estimation of the energy functional on $\bar{H}$ is

$$
I\left(u_{\varepsilon}, v_{\varepsilon}\right)=\sup _{\bar{H}} I .
$$

Remark 2.4 We point out that, in contrast with Theorem 2.3, only constant positive solutions are expected to exist for large values of $\varepsilon[3,15]$.

Remark 2.5 The estimation (2.3) is an important step in the proof of

$$
H\left(P_{0}\right)=\max _{P \in \partial \Omega} H(P),
$$

where $H(P)$ denotes the mean curvature of $\partial \Omega$ at the boundary point $P$. So we can conclude the least energy solutions of system (1.1) concentrate at a point of the boundary, which maximizes the mean curvature of the boundary of $\Omega$.

\section{Proof of Theorem 2.3}

To prove Theorem 2.3, we need the following lemma. Let $u_{\varepsilon}, v_{\varepsilon}$ be the solutions of (1.1), in order to simplify the notations, we define $u:=u_{\varepsilon}, v:=v_{\varepsilon}$,

$$
I_{1}(t):=I((1-t) \phi+t u,(1-t)(-\phi)+t v), \quad \forall \phi \in H^{1}
$$

and $\bar{u}=\phi+t(u-\phi), \bar{v}=-\phi+t(v+\phi)$.

Lemma 3.1 Supposing the assumptions in Theorem 2.3 hold, $I_{1}(t)$ admits the following properties:

(i) $I_{1}^{\prime}(1)=0, I_{1}(0) \leq 0, I_{1}(1)>0$,

(ii) $I_{1} I_{1}(t) \rightarrow-\infty, t \rightarrow \infty$,

(iii) $I_{1}^{\prime \prime}(1)<0$. 
Proof Proof of $(\mathrm{i})_{1}$. By the definition of $I_{1}, I_{1}^{\prime}(t)=I^{\prime}(\bar{u}, \bar{v}) \cdot(u-\phi, v+\phi)$, then $I_{1}^{\prime}(1)=I^{\prime}(u, v)$. $(u-\phi, v+\phi)$, where $u$ and $v$ are the solutions of (1.1), that follows $I_{1}^{\prime}(1)=0$.

Again by (3.1),

$$
\begin{aligned}
I_{1}(0) & =I(\phi,-\phi)=\int_{\Omega}(\langle\nabla \phi, \nabla(-\phi)\rangle+\phi \cdot(-\phi))-\int_{\Omega}(F(\phi)+G(-\phi)) \\
& =-\|\phi\|^{2}-\int_{\Omega}(F(\phi)+G(-\phi)),
\end{aligned}
$$

by $\left(\mathrm{S}_{2}\right)$, we have $F \geq 0, G \geq 0$, so $I_{1}(0) \leq 0$.

Next, we want to show $I_{1}(1)>0$.

$$
\begin{aligned}
I_{1}(1) & =\int_{\Omega}(\langle\nabla u, \nabla v\rangle+u v)-\int_{\Omega}(F(u)+G(v)) \\
& =\int_{\Omega}\left(\frac{1}{2}\langle\nabla u, \nabla v\rangle+\frac{1}{2} u v\right)+\int_{\Omega}\left(\frac{1}{2}\langle\nabla u, \nabla v\rangle+\frac{1}{2} u v\right)-\int_{\Omega}(F(u)+G(v)) \\
& =\frac{1}{2} \int_{\Omega}\langle-\Delta u+u, v\rangle+\frac{1}{2} \int_{\Omega}\langle-\Delta v+v, u\rangle-\int_{\Omega}(F(u)+G(v)) \\
& =\frac{1}{2} \int_{\Omega} g(v) v+\frac{1}{2} \int_{\Omega} f(u) u-\int_{\Omega}(F(u)+G(v)) \\
& =\int_{\Omega}\left(\frac{1}{2} f(u) u-F(u)\right)+\int_{\Omega}\left(\frac{1}{2} g(v) v-G(v)\right) .
\end{aligned}
$$

From $\left(\mathrm{S}_{2}\right)$, we can deduce there exist $\delta>0$, such that $f^{\prime}(t) t \geq(1+\delta) f(t)$, then

$$
\int_{0}^{s} f^{\prime}(t) t \geq(1+\delta) \int_{0}^{s} f(t)=(1+\delta) F(s) .
$$

The left side in last inequality is equal to $f(s) s-F(s)$, so $f(s) s \geq(2+\delta) F(s)$. By the same way, $g(s) s \geq(2+\delta) G(s)$. Then (3.2) can change to

$$
I_{1}(1) \geq\left(\frac{1}{2}-\frac{1}{2+\delta}\right) \int_{\Omega}(f(u) u+g(v) v)>0 .
$$

So, we get the properties (i) .

To prove (ii) $)_{1}$ is equal to show

$$
I(\bar{u}, \bar{v}) \rightarrow-\infty, \quad t \rightarrow \infty
$$

We only need to prove for $2<p<2 N /(N-2)$,

$$
\liminf _{t \rightarrow \infty} \frac{1}{|1-t|^{p}} \int_{\Omega}(F(\bar{u})+G(\bar{v}))>0
$$

By $\left(\mathrm{S}_{1}\right)$, there exist $\mathrm{C}$ such that

$$
\frac{1}{|1-t|^{p}} \int_{\Omega} F((1-t) \phi+t u)=\frac{C}{|1-t|^{p}} \int_{\Omega}|(1-t) \phi+t u|^{p}+o(1) .
$$


As the same,

$$
\frac{1}{|1-t|^{p}} \int_{\Omega} G((t-1) \phi+t v)=\frac{C}{|1-t|^{p}} \int_{\Omega}|(t-1) \phi+t v|^{p}+o(1)
$$

We prove (3.3) by contradiction. Assume that there exists a subsequence $t_{n}$ such that

$$
\frac{1}{\left|1-t_{n}\right|^{p}} \int_{\Omega}\left(F\left(\phi+t_{n}(u-\phi)\right)+G\left(-\phi+t_{n}(v+\phi)\right)\right) \leq 0
$$

that is,

$$
\int_{\Omega}\left(\left|\phi+\frac{t_{n}}{1-t_{n}} u\right|^{p}+\left|-\phi+\frac{t_{n}}{1-t_{n}} v\right|^{p}\right)+o(1)=0 .
$$

So, for any $\phi \in H^{1}(\Omega)$, we have

$$
\int_{\Omega}\left(\left|\phi+\frac{t_{n}}{1-t_{n}} u\right|^{p}+\left|-\phi+\frac{t_{n}}{1-t_{n}} v\right|^{p}\right)=o(1) .
$$

Take $\phi=0$ in the last equality, then

$$
\left|\frac{t_{n}}{1-t_{n}}\right|^{p} \int_{\Omega}\left(u^{p}+v^{p}\right)=o(1) .
$$

For $p$ is a number between 2 and $2 N /(N-2), u \neq \equiv 0$, we deduce $u=-v$. It contradicts with the original assumption of $u \neq-v$.

Now, we turn to the prove of (iii) $)_{1}$. By (3.1),

$$
\begin{aligned}
I_{1}(t)= & \int_{\Omega}(\langle\nabla(\phi+t(u-\phi)), \nabla(-\phi+t(v+\phi))\rangle+(\phi+t(u-\phi)) \cdot(-\phi+t(v+\phi))) \\
& -\int_{\Omega}(F(\bar{u})+G(\bar{v})) \\
= & \int_{\Omega}(\langle\nabla \phi,-\nabla \phi\rangle+\langle\nabla \phi, t \nabla(v+\phi)\rangle+\langle t \nabla(u-\phi),-\nabla \phi\rangle) \\
& +\int_{\Omega}\langle t \nabla(u-\phi), t \nabla(v+\phi)\rangle+\int_{\Omega}(\phi \cdot(-\phi)+t \phi(v+\phi)-t \phi(u-\phi)) \\
& +\int_{\Omega} t^{2}(u-\phi)(v+\phi)-\int_{\Omega}(F(\bar{u})+G(\bar{v})),
\end{aligned}
$$

then

$$
\begin{aligned}
I_{1}^{\prime \prime}(t)= & 2 \int_{\Omega}(\langle\nabla(u-\phi), \nabla(v+\phi)\rangle+(u-\phi)(v+\phi))-\int_{\Omega} f^{\prime}(\bar{u})(u-\phi)^{2} \\
& -\int_{\Omega} g^{\prime}(\bar{v})(v+\phi)^{2} \\
= & 2 \int_{\Omega}(\langle\nabla u, \nabla v\rangle+\langle\nabla u, \nabla \phi\rangle+\langle-\nabla \phi, \nabla v\rangle+\langle-\nabla \phi, \nabla \phi\rangle) \\
& +2 \int_{\Omega}\left(u v+u \phi-\phi v-\phi^{2}\right)-\int_{\Omega} f^{\prime}(\bar{u})(u-\phi)^{2}-\int_{\Omega} g^{\prime}(\bar{v})(v+\phi)^{2}
\end{aligned}
$$




$$
\begin{aligned}
= & \int_{\Omega}((-\Delta v+v) u+(-\Delta u+u) v+2(-\Delta u+u) \phi+2(\Delta v-v) \phi) \\
& +2 \int_{\Omega}\left(-|\nabla \phi|^{2}-\phi^{2}\right)-\int_{\Omega} f^{\prime}(\bar{u})(u-\phi)^{2}-\int_{\Omega} g^{\prime}(\bar{v})(v+\phi)^{2} \\
= & -2\|\phi\|^{2}+\int_{\Omega}(f(u) u+g(v) v+2 g(v) \phi-2 f(u) \phi)-\int_{\Omega} f^{\prime}(\bar{u})(u-\phi)^{2} \\
& -\int_{\Omega} g^{\prime}(\bar{v})(v+\phi)^{2},
\end{aligned}
$$

take $t=1$, then

$$
\begin{aligned}
I_{1}^{\prime \prime}(1)= & -2\|\phi\|^{2}+\int_{\Omega}\left(f(u)(u-2 \phi)-f^{\prime}(u)(u-\phi)^{2}\right)+\int_{\Omega} g(v)(v+2 \phi) \\
& -\int_{\Omega} g^{\prime}(v)(v-(-\phi))^{2} .
\end{aligned}
$$

By $\left(\mathrm{S}_{2}\right)$, for some $\delta>0$,

$$
\int_{\Omega} f(u)(u-2 \phi)-f^{\prime}(u)(u-\phi)^{2} \leq \int_{\Omega}-\delta f(u) u,
$$

in the same way,

$$
\int_{\Omega} g(v)(v+2 \phi)-g^{\prime}(v)(v-(-\phi))^{2} \leq \int_{\Omega}-\delta g(v) v .
$$

Thus, we obtain $I_{1}^{\prime \prime}(1)<0$.

Proposition 3.2 (Theorem 1.1 in [18]) Under assumptions $(\mathrm{H})$, there exists $\varepsilon_{0}>0$ such that for any $0<\varepsilon<\varepsilon_{0}$, problem (1.1) has nonconstant positive solutions $u_{\varepsilon}, v_{\varepsilon} \in C^{2}(\Omega)$. Moreover, both functions $u_{\varepsilon}$ and $v_{\varepsilon}$ attain their maximum value at some unique and common point $P_{\varepsilon} \in \partial \Omega$. (The assumption $(\mathrm{H})$ is composed of $\left(\mathrm{S}_{1}\right),\left(\mathrm{S}_{3}\right)$ and the following (3.5).)

Remark 3.3 We will compare our assumptions $\left(\mathrm{S}_{1}\right)-\left(\mathrm{S}_{3}\right)$ with the conditions $(\mathrm{H})$ of Proposition 3.2 in the following proof of Theorem 2.3.

Proof of Theorem 2.3 The existence of solutions of (1.1) can follow the steps of Theorem 1.1 in [18]. They use some ideas introduced by Del Pino and Felmer [6], and differ from the method of $\mathrm{Ni}$ and Takagi. It needs to be pointed out that $\left(\mathrm{S}_{2}\right)$ implies the following conditions:

For some $\delta>0, s \in \mathbb{R}, s \neq 0$,

$$
f(s) s \geq(2+\delta) F(s)>0, \quad g(s) s \geq(2+\delta) G(s)>0 .
$$

The assumption $(\mathrm{H})$ in Proposition 3.2 is composed of $\left(\mathrm{S}_{1}\right),\left(\mathrm{S}_{3}\right)$ and (3.5). By Proposition 3.2, the existence of solutions can be proved under $(\mathrm{H})$. So, we can get the existence of solutions of (1.1) under $\left(\mathrm{S}_{1}\right)-\left(\mathrm{S}_{3}\right)$.

The rest of the paper is devoted to the proof of (2.3). By the definition of space $\bar{H}$, we only need to prove for any $\phi \in H^{1}(\Omega), t \in \mathbb{R}, t>0$ the following holds,

$$
I(\phi+t u,-\phi+t v) \leq I(u, v) .
$$


Obviously $t \not \equiv 1$. By (3.1), (3.6) is equal to

$$
I_{1}(t) \leq I_{1}(1)
$$

We prove (3.7) by contradiction, suppose that the maximum point of $I_{1}(t)$ is $t_{0} \geq 0$, and $t_{0} \neq 1$.

$$
\begin{aligned}
I_{1}^{\prime}(t)= & \int_{\Omega}(\langle\nabla \phi, \nabla(v+\phi)\rangle+\langle\nabla(u-\phi),-\nabla \phi\rangle+2 t\langle\nabla(u-\phi), \nabla(v+\phi)\rangle) \\
& +\int_{\Omega}(\phi(v+\phi)-\phi(u-\phi)+2 t(u-\phi)(v+\phi))-\int_{\Omega} f(\bar{u})(u-\phi) \\
& -\int_{\Omega} g(\bar{v})(v+\phi) \\
= & \int_{\Omega}(\langle\nabla(\phi+t(u-\phi)), \nabla(v+\phi)\rangle+\langle\nabla(u-\phi), \nabla(-\phi+t(v+\phi))\rangle) \\
& +\int_{\Omega}((u-\phi)(-\phi+t(v+\phi))+(v+\phi)(\phi+t(u-\phi)))-\int_{\Omega} f(\bar{u})(u-\phi) \\
& -\int_{\Omega} g(\bar{v})(v+\phi) \\
= & \int_{\Omega}(\langle\nabla \bar{u}, \nabla(v+\phi)\rangle+\langle\nabla(u-\phi), \nabla \bar{v}\rangle+(u-\phi) \cdot \bar{v}+(v+\phi) \cdot \bar{u}) \\
& -\int_{\Omega}(f(\bar{u})(u-\phi)+g(\bar{v})(v+\phi)) .
\end{aligned}
$$

Let $I_{1}^{\prime}(t)=0$, we get

$$
\begin{aligned}
& \int_{\Omega}(\langle\nabla \bar{u}, \nabla(v+\phi)\rangle+\langle\nabla(u-\phi), \nabla \bar{v}\rangle+(u-\phi) \cdot \bar{v}+(v+\phi) \cdot \bar{u}) \\
& =\int_{\Omega}(f(\bar{u})(u-\phi)+g(\bar{v})(v+\phi)) .
\end{aligned}
$$

Thus, by (3.8), we have

$$
\begin{aligned}
2 I(\bar{u}, \bar{v})= & \int_{\Omega}(\langle\nabla \bar{u}, \nabla(-\phi+t(v+\phi))\rangle+\bar{u} \cdot(-\phi+t(v+\phi)) \\
& +\langle\nabla(\phi+t(u-\phi)), \nabla \bar{v}\rangle)+\int_{\Omega}(\phi+t(u-\phi)) \cdot \bar{v}-2 \int_{\Omega}(F(\bar{u})+G(\bar{v})) \\
= & \int_{\Omega}(\langle\nabla \bar{u}, \nabla(-\phi)\rangle+\bar{u} \cdot(-\phi)+\langle\nabla \phi, \nabla \bar{v}\rangle+\phi \cdot \bar{v})+\int_{\Omega}\langle\nabla \bar{u}, t \nabla(v+\phi)\rangle \\
& +\int_{\Omega}(\bar{u} \cdot t(v+\phi)+\langle t \nabla(u-\phi), \nabla \bar{v}\rangle+t(u-\phi) \cdot \bar{v})-2 \int_{\Omega}(F(\bar{u})+G(\bar{v})) \\
= & \int_{\Omega}(\langle\nabla \bar{u}, \nabla(-\phi)\rangle+\bar{u} \cdot(-\phi)+\langle\nabla \phi, \nabla \bar{v}\rangle+\phi \cdot \bar{v})+\int_{\Omega} f(\bar{u}) \cdot t \cdot(u-\phi) \\
& +\int_{\Omega} g(\bar{v}) \cdot t \cdot(v+\phi)-2 \int_{\Omega}(F(\bar{u})+G(\bar{v}))=: 2 I_{2}(t) .
\end{aligned}
$$

We claim that the function $I_{2}(t)$, defined in (3.9), has the following properties: 
(i) $I_{2}(0) \leq 0, I_{2}(1)>0, I_{2}^{\prime}(1)>0$;

(ii) $)_{2}$ For any $t \geq 0$, if $I_{2}^{\prime}(t)=0$, then $I_{2}(t) \leq 0$;

(iii) $I_{2}(t) \rightarrow-\infty$, as $t \rightarrow \infty$.

First, we prove (i) $)_{2}$. In fact, by (3.1) and (3.9),

$$
\begin{aligned}
I_{2}(0) & =\frac{1}{2}\left\{\int_{\Omega} 2(\langle\nabla \phi, \nabla(-\phi)\rangle+\phi \cdot(-\phi))-2 \int_{\Omega}(F(\phi)+G(-\phi))\right\} \\
& =-\|\phi\|^{2}-\int_{\Omega}(F(\phi)+G(-\phi)) .
\end{aligned}
$$

Following from assumption $\left(\mathrm{S}_{2}\right)$, we obtain $I_{2}(0) \leq 0$.

Again by (3.9) and Lemma 3.1, $I_{2}(1)=I_{1}(1)$ and $I_{1}(1)>0$, so $I_{2}(1)>0$. Next, we want to compute $I_{2}^{\prime}(1)$. From (3.9),

$$
\begin{aligned}
2 I_{2}(t)= & \int_{\Omega}(\langle\nabla \phi, \nabla(-\phi)\rangle+\langle\nabla(t(u-\phi)), \nabla(-\phi)\rangle+\phi \cdot(-\phi)+t(u-\phi)(-\phi)) \\
& +\int_{\Omega}(\langle\nabla \phi, \nabla(-\phi)\rangle+\langle\nabla \phi, \nabla(t(v+\phi))\rangle+\phi \cdot(-\phi)+\phi \cdot t \cdot(v+\phi)) \\
& +\int_{\Omega}(f(\bar{u}) \cdot t \cdot(u-\phi)+g(\bar{v}) \cdot t \cdot(v+\phi))-2 \int_{\Omega}(F(\bar{u})+G(\bar{v})), \\
2 I_{2}^{\prime}(t)= & \int_{\Omega}(\langle\nabla(u-\phi), \nabla(-\phi)\rangle+(u-\phi)(-\phi))+\int_{\Omega}(\langle\nabla \phi, \nabla(v+\phi)\rangle+\phi(v+\phi)) \\
& +\int_{\Omega}\left(f^{\prime}(\bar{u}) \cdot t \cdot(u-\phi)^{2}-f(\bar{u})(u-\phi)+g^{\prime}(\bar{v}) \cdot t \cdot(v+\phi)^{2}-g(\bar{v})(v+\phi)\right) .
\end{aligned}
$$

Then

$$
\begin{aligned}
2 I_{2}^{\prime}(1)= & \int_{\Omega}(\langle\nabla(u-\phi), \nabla(-\phi)\rangle+(u-\phi)(-\phi))+\int_{\Omega}(\langle\nabla \phi, \nabla(v+\phi)\rangle+\phi(v+\phi)) \\
& +\int_{\Omega}\left(f^{\prime}(u)(u-\phi)^{2}-f(u)(u-\phi)+g^{\prime}(v)(v+\phi)^{2}-g(v)(v+\phi)\right) \\
= & 2\|\phi\|^{2}+\int_{\Omega}(\langle-\Delta u,-\phi\rangle+u(-\phi)+\langle-\Delta v, \phi\rangle+v \phi)+\int_{\Omega}(f(u) \phi+g(v)(-\phi)) \\
& +\int_{\Omega}\left(f^{\prime}(u)(u-\phi)^{2}+g^{\prime}(v)(v+\phi)^{2}-f(u) u-g(v) v\right) \\
= & 2\|\phi\|^{2}+2 \int_{\Omega}(f(u) \phi+g(v)(-\phi))+\int_{\Omega}\left(f^{\prime}(u)(u-\phi)^{2}+g^{\prime}(v)(v+\phi)^{2}\right) \\
& -\int_{\Omega}(f(u) u+g(v) v) .
\end{aligned}
$$

Combined with (3.4), $2 I_{2}^{\prime}(1)=-I_{1}^{\prime \prime}(1)$. By Lemma 3.1, $I_{1}^{\prime \prime}(1)<0$, so $I_{2}^{\prime}(1)>0$.

Now, we turn to the proof of (ii) $)_{2}$. Let $I_{2}^{\prime}(t)=0$, by (3.11),

$$
\begin{aligned}
& t \int_{\Omega}(\langle\nabla(u-\phi), \nabla(-\phi)\rangle+(u-\phi)(-\phi))+t \int_{\Omega}(\langle\nabla \phi, \nabla(v+\phi)\rangle+\phi(v+\phi)) \\
& \quad=-\int_{\Omega}\left(f^{\prime}(\bar{u}) \cdot t^{2} \cdot(u-\phi)^{2}-t f(\bar{u})(u-\phi)+g^{\prime}(\bar{v}) \cdot t^{2} \cdot(v+\phi)^{2}-t g(\bar{v})(v+\phi)\right) .
\end{aligned}
$$


Then by (3.10),

$$
\begin{aligned}
2 I_{2}(t)= & -\|\phi\|^{2}-\int_{\Omega}\left(f^{\prime}(\bar{u}) \cdot t^{2} \cdot(u-\phi)^{2}-2 t f(\bar{u})(u-\phi)+2 F(\bar{u})\right) \\
& -\int_{\Omega}\left(g^{\prime}(\bar{v}) \cdot t^{2} \cdot(v+\phi)^{2}-2 \operatorname{tg}(\bar{v})(v+\phi)+2 G(\bar{v})\right) .
\end{aligned}
$$

By $\left(S_{3}\right)$, we get

$$
\begin{aligned}
f^{\prime}(\bar{u}) \cdot t^{2} \cdot(u-\phi)^{2}+2 F(\bar{u}) & \geq 2 t(u-\phi) \sqrt{2 f^{\prime}(\bar{u}) F(\bar{u})} \\
& \geq 2 t(u-\phi) f(\bar{u}),
\end{aligned}
$$

similarly,

$$
g^{\prime}(\bar{v}) \cdot t^{2} \cdot(v+\phi)^{2}+2 G(\bar{v}) \geq 2 t(v+\phi) g(\bar{v}),
$$

then by (3.12), $I_{2}(t) \leq 0$. We proved the property (ii) $)_{2}$.

The proof of (iii) $)_{2}$ is similar to (ii) $)_{1}$. Then we complete the proof of the claim.

Suppose that the maximum point of $I_{1}(t)$ is $t_{0} \geq 0, t_{0} \neq 1$, then either $t_{0} \in(0,1)$ or $t_{0} \in$ $(1,+\infty)$. If $t_{0} \in(0,1), I_{1}\left(t_{0}\right)>I_{1}(1)$, by (3.1), (3.9) and property (i) $)_{2}$ of $I_{2}, I_{2}\left(t_{0}\right)>I_{2}(1)>0$, $I_{2}(0) \leq 0$. By the property (iii) $)_{2}$ of $I_{2}$, there exist $\hat{t}>0$, such that

$$
I_{2}^{\prime}(\hat{t})=0 \quad \text { and } \quad I_{2}(\hat{t})>0 .
$$

If $t_{0} \in(1,+\infty)$, then $I_{1}\left(t_{0}\right)>I_{1}(1)$. By Lemma 3.1, $I_{1}^{\prime}(1)=0, I_{1}^{\prime \prime}(1)<0$, so there exist $t_{1} \in$ $\left(1, t_{0}\right)$, such that $I_{1}\left(t_{1}\right)<I_{1}(1)$, and $I_{1}^{\prime}\left(t_{1}\right)=0$. Thus, $I_{2}\left(t_{1}\right)<I_{2}(1) . I_{2}^{\prime}(1)>0$, so there exist $\tilde{t} \in\left(1, t_{1}\right)$, such that

$$
I_{2}^{\prime}(\tilde{t})=0 \quad \text { and } \quad I_{2}(\tilde{t})>0 .
$$

In fact, (3.13) and (3.14) is a contradiction to the nature (ii) $)_{2}$ of $I_{2}(t)$, that is, for any $t \geq 0$, if $I_{2}^{\prime}(t)=0$, the value of $I_{2}(t)$ must be smaller than 0 .

Having reached a contradiction, this completes the proof of Theorem 2.3.

\section{Competing interests}

There are no financial competing interests in this manuscript. There are no non-financial competing interests (political, personal, religious, ideological, academic, intellectual, commercial or any other) to declare in relation to this manuscript.

\section{Authors' contributions}

There is only one author in the manuscript. JZ designed the study. She carried out the studies, and drafted the manuscript. The author read and approved the final manuscript.

\section{Acknowledgements}

The author is supported by the project of 'Youth Innovation', funded by the Department of Science and Technology of Fujian province (2011 J05003), and supported by the Projects A of the Educational Department of Fujian Province (JA11053). 


\section{References}

1. Gierer, A, Meinhardt, H: A theory of biological pattern formation. Kybernetik 12, 30-39 (1972)

2. Lin, CS, Ni, WM, Takagi, I: Large amplitude stationary solutions to a chemotaxis system. J. Differential Equations 72 , 1-27 (1988)

3. Ni, WM, Takagi, I: On the Neumann problem for some semilinear elliptic equations and systems of activator-inhibitor type. Trans. Amer. Math. Soc. 297, 351-368 (1986)

4. Ni, WM, Takagi, I: On the shape of least-energy solutions to a semilinear Neumann problem. Comm. Pure Appl. Math. 44, 819-851 (1991)

5. Ni, WM, Takagi, I: Locating peaks of least-energy solutions to a semilinear Neumann problem. Duke Math. J. 70, 247-281 (1993)

6. Del Pino, M, Felmer, P: Spike-layered solutions of singularly perturbed elliptic problems in a degenerate setting Indiana Univ. Math. J. 48, 883-898 (1999)

7. $\mathrm{Ni}, \mathrm{WM}, \mathrm{Wei}, \mathrm{J}$ : On the location and profile of spike-layer solutions to singularly perturbed semilinear Dirichlet problems. Comm. Pure Appl. Math. 48, 731-768 (1995)

8. Wang, ZQ: On the existence of multiple single-peaked solution for a semilinear Neumann problem. Arch. Ration. Mech. Anal. 120, 375-399 (1992)

9. Grossi, M, Pistoia, A, Wei, J: Existence of multipeak solutions for a semilinear Neumann problem via nonsmooth critical point theory. Calc. Var. Partial Differential Equations 11, 143-175 (2000)

10. Grossi, M, Pistoia, A: On the effect of critical points of distance function in superlinear elliptic problems. Adv. Differential Equ 5, 1397-1420 (2000)

11. Li, YY, Nirenberg, L: The Dirichlet problem for singularly perturbed elliptic equations. Comm. Pure Appl. Math. 51, 1445-1490 (1998)

12. Wei, J: On the boundary spike layer solutions to a singularly perturbed Neumann problem. J. Differential Equations $134,104-133$ (1997)

13. Wei, J: On the interior spike layer solutions of a singularly perturbed semilinear Neumann problem. Tohoku Math. J. 50, 159-178 (1998)

14. Wei, J: On the interior spike solutions for some singular perturbation problems. Proc. Roy. Soc. Edinb. Sect A 128, 849-874 (1998)

15. Avila, Al, Yang, J: On the existence and shape of least energy solutions for some elliptic systems. J. Differentia Equations 191, 348-376 (2003)

16. Busca, J, Sirakov, B: Symmetry results for semilinear elliptic systems in the whole space. J. Differential Equations 163 41-56 (2000)

17. Clement, P, De Figueiredo, DG, Mitidieri, E: Positive solutions of semilinear elliptic systems. Comm. Partial Differential Equations 17, 923-940 (1992)

18. Pistoia, A, Ramos, M: Locating the peaks of the least energy solutions to an elliptic system with Neumann boundary conditions. J. Differential Equations 201, 160-176 (2004)

19. Pistoia, A, Ramos, M: Locating the peaks of the least energy solutions to an elliptic system with Dirichlet boundary conditions. Nonlinear Differ. Equ. Appl. 15, 1-23 (2008)

20. Gidas, B, Ni, WM, Nirenberg, L: Symmetry of positive solutions of non-linear elliptic equations in $\mathbb{R}^{n}$. In: Mathematical Analysis and Applications. Part A. Adv. in Math. Suppl. Stud., vol. 7, pp. 369-402 (1981)

21. Abbondandolo, A, Felmer, P, Molina, J: An estimate on the relative Morse index for strongly indefinite functionals. Electron. J. Differ. Equ. Conf. 6, 1-11 (2001)

22. Ambrosetti, A, Rabinowitz, PH: Dual variational methods in critical point theory. J. Funct. Anal. 14, 349-381 (1973)

doi:10.1186/1687-2770-2013-194

Cite this article as: Zeng: The estimates on the energy functional of an elliptic system with Neumann boundary conditions. Boundary Value Problems 2013 2013:194.

\section{Submit your manuscript to a SpringerOpen ${ }^{\circ}$ journal and benefit from:}

- Convenient online submission

- Rigorous peer review

Immediate publication on acceptance

- Open access: articles freely available online

- High visibility within the field

- Retaining the copyright to your article 